Journal of Mathematics and Informatics

Vol. 6, 2016, 53-66

ISSN: 2349-0632 (P), 2349-0640 (online)

Published 30 November 2016

www.researchmathsci.org

DOI: http://dx.doi.org/10.22457/jmi.v6a7

Journal of

Mathematics and

Informatics

\title{
Interval Valued Fuzzy Prime Bi-ideals and Interval Valued Fuzzy Strongly Prime Bi-ideals in Near-rings
}

\author{
V. Vetrivel ${ }^{1}$ and $P$. Murugadas ${ }^{2}$
}

Department of Mathematics, Annamalai University, Annamalainagar-608002, India

Emil: ${ }^{1}$ vetrivelmath@gmail.com; ${ }^{2}$ bodi_muruga@yahoo.com

*Corresponding author. E-mail address: bodi_muruga@yahoo.com

Received 23 September 2016; accepted 17 October 2016

Abstract. In this paper, prime, strongly prime, irreducible and strongly irreducible ideals of an interval valued fuzzy bi-ideals are defined and some results are explored.

Keywords: Interval valued fuzzy Near-ring, Interval valued fuzzy prime ideals, Interval valued fuzzy irreducible ideals.

\section{AMS Mathematics Subject Classification (2010): 16A72, 94D05}

\section{Introduction}

After the introduction of fuzzy set by Zadeh [9] there have been a number of generalizations of this fundamental concept. In 1975, Zadeh [10] introduced the concept of interval valued fuzzy subset, where the values of the membership functions are intervals of numbers instead of the numbers. The fuzzy algebraic structures play a prominent role in mathematics with wide applications in many other branches such as theoretical physics, computer sciences, control engineering, information sciences, coding theory, topological spaces, logic, set theory, group theory, groupoids, real analysis, measure theory etc.

In 1971, Rosenfeld [5] introduced fuzzy subgroup and introduced some of its properties. Abou-Zaid [2] in 1991, initiated study on ideals of fuzzy subnear-rings, evaluated fuzzy left (right) ideals of near-rings and discovered some prominent characteristics of fuzzy prime ideals of a near-rings. Jun and Kim [4] and Dawaz [3] applied a few concepts of fuzzy ideals and i-v fuzzy ideals in near-rings.

The notion of bi-ideals in near-rings was brought forth by Chelvam and Ganesan [8]. Abbassi and Rizvi [1] meditated and research upon the prime ideals in near-rings in 2008. In this discourse, we have thoroughly studied i-v fuzzy prime bi-ideals, i-v fuzzy semiprime bi-ideals, i-v fuzzy reducible and i-v fuzzy irreducible bi-ideals in near-rings.

\section{Preliminaries}

Definition 2.1. [4] A non-empty set $N$ with two binary operations " +" and "." is called a near-ring if

(1) $(N,+)$ is a group,

(2) $(N,$.$) is a semigroup,$ 


\section{V.Vetrivel and P.Murugadas}

(3) $x \cdot(y+z)=x \cdot y+x \cdot z$, for all $x, y, z \in N$.

We use word 'near-ring' to mean 'left near-ring'. We denote xy instead of x.y. Note that $x 0=0$ and $x(-y)=-x y$ but in general $0 x \neq 0$ for some $x \in N$.

Definition 2.2. [4] An ideal $I$ of a near-ring $N$ is a subset of $N$ such that

(4) $(I,+)$ is a normal subgroup of $(N,+)$,

(5) $N I \subseteq I$,

(6) $((x+i) y-x y) \in I$ for any $i \in I$ and $x, y \in N$.

Note that $I$ is a left ideal of $N$ if $I$ satisfies (4) and (5), and $I$ is a right ideal of $N$ if $I$ satisfies (4) and (6).

Definition 2.3. [10] A two sided $N$-subgroup of a near-ring $N$ is a subset $H$ of $N$ such that

(i) $(H,+)$ is a subgroup of $(N,+)$,

(ii) $N H \subset H$,

(iii) $H N \subset H$.

If $H$ satisfies (i) and (ii) then it is called a left $N$-subgroup of $N$. If $H$ satisfies (i) and (iii) then it is called a right $N$ - subgroup of $N$.

Definition 2.4. [4] Let $N$ be a near-ring. Given two subsets $A$ and $B$ of $N$, the product $A B=\{a b \mid a \in A, b \in B\}$ Also we define another operation ' $*$ ' on the class of subsets of $N$ given by $A * B=\left\{a\left(a^{\prime}+b\right)-a a^{\prime} \mid a, a^{\prime} \in A, b \in B\right\}$.

Definition 2.5. [10] An interval number $\bar{a}$ on $[0,1]$ is a closed subinterval of $[0,1]$, that is, $\bar{a}=\left[a^{-}, a^{+}\right]$such that $0 \leq a^{-} \leq a^{+} \leq 1$, where $a^{-}$and $a^{+}$are the lower and upper end limits of $\bar{a}$ respectively. The set of all closed subintervals of $[0,1]$ is denoted by $\mathrm{D}[0,1]$. We also identify the interval $[\mathrm{a}, \mathrm{a}]$ by the number $a \in[0,1]$. For any interval numbers $\bar{a}_{i}=\left[a_{i}^{-}, a_{i}^{+}\right], \bar{b}_{i}=\left[b_{i}^{-}, b_{i}^{+}\right] \in D[0,1], i \in I$, we define $\max ^{i}\left\{\bar{a}_{i}, \bar{b}_{i}\right\}=\left[\max ^{i}\left\{a_{i}^{-}, b_{i}^{-}\right\}, \max ^{i}\left\{a_{i}^{+}, b_{i}^{+}\right\}\right]$, $\min ^{i}\left\{\bar{a}_{i}, \bar{b}_{i}\right\}=\left[\min ^{i}\left\{a_{i}^{-}, b_{i}^{-}\right\}, \min ^{i}\left\{a_{i}^{+}, b_{i}^{+}\right\}\right]$, $\inf ^{i} \bar{a}_{i}=\left[\bigcap_{i \in I} a_{i}^{-}, \bigcap_{i \in I} a_{i}^{+}\right], \sup ^{i} \bar{a}_{i}=\left[\bigcup_{i \in I} a_{i}^{-}, \bigcup_{i \in I} a_{i}^{+}\right]$

In this notation $\overline{0}=[0,0]$ and $\overline{1}=[1,1]$. For any interval numbers $\bar{a}=\left[a^{-}, a^{+}\right]$and $\bar{b}=\left[b^{-}, b^{+}\right]$on $[0,1]$, define

(1) $\bar{a} \leq \bar{b}$ if and only if $a^{-} \leq b^{-}$and $a^{+} \leq b^{+}$.

(2) $\bar{a}=\bar{b}$ if and only if $a^{-}=b^{-}$and $a^{+}=b^{+}$.

(3) $\bar{a}<\bar{b}$ if and only if $\bar{a} \leq \bar{b}$ and $\bar{a} \neq \bar{b}$

(4) $k \bar{a}=\left[k a^{-}, k a^{+}\right]$, whenever $0 \leq k \leq 1$. 
Interval Valued Fuzzy Prime Bi-Ideals and Interval Valued Fuzzy Strongly Prime BiIdeals in Near-Rings

Definition 2.6. [10] Let $X$ be any set. A mapping $\bar{A}: X \rightarrow D[0,1]$ is called an interval-valued fuzzy subset (briefly, i-v fuzzy subset) of $X$ where $\mathrm{D}[0,1]$ denotes the family of all closed subintervals of $[0,1]$ and $\bar{A}(x)=\left[A^{-}(x), A^{+}(x)\right]$ for all $x \in X$, where $A^{-}$and $A^{+}$are fuzzy subsets of $X$ such that $A^{-}(x) \leq A^{+}(x)$ for all $x \in X$.

Note that $\bar{A}(x)$ is an interval (a closed subset of $[0,1])$ and not a number from the interval $[0,1]$ as in the case of fuzzy subset.

Definition 2.7. [10] A mapping $\min ^{i}: D[0,1] \times D[0,1] \rightarrow D[0,1]$ defined by $\min ^{i}(\bar{a}, \bar{b})=\min \left\{a^{-}, b^{-}\right\}, \min \left\{a^{+}, b^{+}\right\}$for all $\bar{a}, \bar{b} \in D[0,1]$ is called an interval minnorm.

Definition 2.8.[10] A mapping $\max ^{i}: D[0,1] \times D[0,1] \rightarrow D[0,1]$ defined by $\max ^{i}(\bar{a}, \bar{b})=\left[\max \left\{a^{-}, b^{-}\right\}, \max \left\{a^{+}, b^{+}\right\}\right]$for all $\bar{a}, \bar{b} \in D[0,1]$ is called an interval max-norm.

Let $\min ^{i}$ and $\max ^{i}$ be the interval min-norm and max-norm on $\mathrm{D}[0,1]$ respectively. Then the following are true.

1. $\min ^{i}\{\bar{a}, \bar{a}\}=\bar{a}$ and $\max ^{i}\{\bar{a}, \bar{a}\}=\bar{a}$ for all $\bar{a} \in D[0,1]$.

2. $\min ^{i}\{\bar{a}, \bar{b}\}=\min ^{i}\{\bar{b}, \bar{a}\}$ and $\max ^{i}\{\bar{a}, \bar{b}\}=\max ^{i}\{\bar{b}, \bar{a}\}$ for all $\bar{a}, \bar{b} \in D[0,1]$.

3. If $\bar{a} \geq \bar{b} \in D[0,1]$, then $\min ^{i}\{\bar{a}, \bar{c}\} \geq \min ^{i}\{\bar{b}, \bar{c}\}$ and $\max ^{i}\{\bar{a}, \bar{c}\} \geq \max ^{i}\{\bar{b}, \bar{c}\}$ for all $\bar{c} \in D[0,1]$.

Definition 2.9. [10] Let $\bar{A}$ be an i-v fuzzy subset of a set $X$ and $\left[t_{1}, t_{2}\right] \in D[0,1]$, then the set $\bar{U}\left(\bar{A}:\left[t_{1}, t_{2}\right]\right)=\left\{x \in X \mid \bar{A}(x) \geq\left[t_{1}, t_{2}\right]\right\}$ is called the upper level set of $\bar{A}$.

Note that

$\bar{U}\left(\bar{A}:\left[t_{1}, t_{2}\right]\right)=\left\{x \in X \mid\left[A^{-}(x), A^{+}(x)\right] \geq\left[t_{1}, t_{2}\right]\right\}$

$=\left\{x \in X \mid A^{-}(x) \geq t_{1}\right\} \cap\left\{x \in X \mid A^{+}(x) \geq t_{2}\right\}$

$=\left(U\left(A^{-}: t_{1}\right)\right) \cap\left(U\left(A^{+}: t_{2}\right)\right)$.

Definition 2.10.[1] If $\bar{\lambda}$ and $\bar{\mu}$ are i-v fuzzy subsets of a near-ring $N$. Then $\bar{\lambda} \cap \bar{\mu}, \bar{\lambda} \cup \bar{\mu}, \bar{\lambda}+\bar{\mu}, \bar{\lambda} \bar{\mu}$ and $\bar{\lambda} * \bar{\mu}$ are i-v fuzzy subsets of $N$ defined by, $(\bar{\lambda} \cap \bar{\mu})(x)=\min ^{i}\{\bar{\lambda}(x), \bar{\mu}(x)\}$ $(\bar{\lambda} \cup \bar{\mu})(x)=\max ^{i}\{\bar{\lambda}(x), \bar{\mu}(x)\}$ 
V.Vetrivel and P.Murugadas

$$
\begin{aligned}
& (\bar{\lambda}+\bar{\mu})(x)= \begin{cases}\sup _{x=y+z}^{i}\left\{\min ^{i}\{\bar{\lambda}(y), \bar{\mu}(z)\}\right\} & \text { if } \mathrm{x} \text { is expressible as } \mathrm{x}=\mathrm{y}+\mathrm{z}, \\
\overline{0} & \text { otherwise. }\end{cases} \\
& (\bar{\lambda} \bar{\mu})(x)= \begin{cases}\sup _{x=y z}^{i}\left\{\min ^{i}\{\bar{\lambda}(y), \bar{\mu}(z)\}\right\} & \text { if } \mathrm{x} \text { is expressible as } \mathrm{x}=\mathrm{yz}, \\
\overline{0} & \text { otherwise. }\end{cases} \\
& (\bar{\lambda} * \bar{\mu})(x)= \begin{cases}\sup _{x=a(b+c)-a b}^{i}\left\{\min ^{i}\{\bar{\lambda}(a), \bar{\mu}(c)\}\right\} & \text { if } \mathrm{x} \text { is expressible as } \mathrm{x}=\mathrm{a}(\mathrm{b}+\mathrm{c})-\mathrm{ab} \\
\overline{0} & \text { otherwise. }\end{cases}
\end{aligned}
$$

Definition 2.11. [1] Let $\bar{\lambda}$ be an i-v fuzzy subset of $N$, then $\bar{\lambda}$ is called an i-v fuzzy left(right) $N$ - subgroup of $N$ if for all $x, y \in N$.

(1) $\bar{\lambda}(x-y) \geq \min ^{i}\{\bar{\lambda}(x), \bar{\lambda}(y)\}$

(2) $\bar{\lambda}(x y) \geq \bar{\lambda}(y),(\bar{\lambda}(x y) \geq \bar{\lambda}(x))$

If $\bar{\lambda}$ is both left and right i-v fuzzy $N$-subgroup of $N$, then it is called an i-v fuzzy $N$ subgroup of $N$.

Definition 2.12. [1] Let $N$ be a near-ring and $\bar{\lambda}$ be i-v fuzzy subset of $N$. We say $\bar{\lambda}$ an i-v fuzzy subnear-ring of $N$ if

(1) $\bar{\lambda}(x-y) \geq \min ^{i}\{\bar{\lambda}(x), \bar{\lambda}(y)\}$

(2) $\bar{\lambda}(x y) \geq \min ^{i}\{\bar{\lambda}(x), \bar{\lambda}(y)\}$ for all $x, y \in N$.

Definition 2.13. [1] Let $\bar{\lambda}$ be a non-empty i-v fuzzy subset of $N . \bar{\lambda}$ is an i-v fuzzy ideal of $N$ if for all $x, y, i \in N$ and

(1) $\bar{\lambda}(x-y) \geq \min ^{i}\{\bar{\lambda}(x), \bar{\lambda}(y)\}$

(2) $\bar{\lambda}(x)=\bar{\lambda}(y+x-y)$

(3) $\bar{\lambda}(x y) \geq \bar{\lambda}(x)$

(4) $(\bar{\lambda}(x(y+i)-x y) \geq \bar{\lambda}(i))$ for any $x, y, i \in N$.

If $\bar{\lambda}$ satisfies (1), (2) and (3), then it is called an i-v fuzzy right ideal of $N$. If $\bar{\lambda}$ satisfies (1), (2) and (4), then it is called an i-v fuzzy left ideal of $N$. If $\bar{\lambda}$ is both i-v fuzzy right as well as i-v fuzzy left ideal of $N$, then $\bar{\lambda}$ is called an i-v fuzzy ideal of $N$.

\section{Example 2.14.}

Let $N=\{a, b, c, d\}$ be a set with two binary operations as follows, 
Interval Valued Fuzzy Prime Bi-Ideals and Interval Valued Fuzzy Strongly Prime BiIdeals in Near-Rings

\begin{tabular}{|c|c|c|c|c|}
\hline+ & $\mathrm{a}$ & $\mathrm{b}$ & $\mathrm{c}$ & $\mathrm{d}$ \\
\hline $\mathrm{A}$ & $\mathrm{a}$ & $\mathrm{b}$ & $\mathrm{c}$ & $\mathrm{d}$ \\
\hline B & $\mathrm{b}$ & $\mathrm{a}$ & $\mathrm{d}$ & $\mathrm{c}$ \\
\hline C & $\mathrm{c}$ & $\mathrm{d}$ & $\mathrm{b}$ & $\mathrm{a}$ \\
\hline D & $\mathrm{d}$ & $\mathrm{c}$ & $\mathrm{a}$ & $\mathrm{b}$ \\
\hline
\end{tabular}

\begin{tabular}{|c|c|c|c|c|}
\hline$\cdot$ & $\mathrm{a}$ & $\mathrm{b}$ & $\mathrm{c}$ & $\mathrm{d}$ \\
\hline $\mathrm{a}$ & $\mathrm{a}$ & $\mathrm{a}$ & $\mathrm{a}$ & $\mathrm{a}$ \\
\hline $\mathrm{b}$ & $\mathrm{a}$ & $\mathrm{a}$ & $\mathrm{a}$ & $\mathrm{a}$ \\
\hline $\mathrm{c}$ & $\mathrm{a}$ & $\mathrm{a}$ & $\mathrm{a}$ & $\mathrm{a}$ \\
\hline $\mathrm{d}$ & $\mathrm{a}$ & $\mathrm{a}$ & $\mathrm{b}$ & $\mathrm{b}$ \\
\hline
\end{tabular}

Then $(N,+,$.$) is a (left) near-ring.$

Define a fuzzy subset $\bar{\lambda}: N \rightarrow[0,1]$ by

$\bar{\lambda}(a)=[1,1], \bar{\lambda}(b)=[0.7,0.8], \bar{\lambda}(d)=\bar{\lambda}(c)=[0.6,0.7]$

Then $\bar{\lambda}$ is an i-v fuzzy ideal of $N$.

Definition 2.15. [4] Let $I$ be a non-empty i-v fuzzy subset of $N$, then the characteristic function of $I$ is denoted by $\bar{\lambda}_{I}$ and is defined as:

$$
\bar{\lambda}_{I}(x)= \begin{cases}\overline{1} & \text { if } \mathrm{x} \in \mathrm{I}, \\ \overline{0} & \text { otherwise }\end{cases}
$$

\section{Some results}

Lemma 3.1. Let $I$ be a subset of $N$. Then $I$ is a left (right) ideal of $N$ if and only if $\bar{\lambda}_{I}$ is an i-v fuzzy left (right) ideal of $N$.

Proof: Let $I$ be a left ideal of $N$.

Let $x, y \in N$.

Suppose that $\bar{\lambda}_{I}(x-y) \leq \min ^{i}\left\{\bar{\lambda}_{I}(x), \bar{\lambda}_{I}(y)\right\}$

$\Rightarrow \bar{\lambda}_{I}(x)=\overline{1}, \bar{\lambda}_{I}(y)=\overline{1}$

and $\bar{\lambda}_{I}(x-y)=\overline{0}$

$\Rightarrow x, y \in I$ and $x-y \notin I$.

This is a contradiction to the fact that $I$ is a left ideal.

Therefore, $\bar{\lambda}_{I}(x-y) \geq \min ^{i}\left\{\bar{\lambda}_{I}(x), \bar{\lambda}_{I}(y)\right\}$.

If $\bar{\lambda}_{I} \neq \bar{\lambda}_{I}(y+x-y)$

If $\bar{\lambda}_{I}(x)=\overline{1}$ and $\bar{\lambda}_{I}(y+x-y)=\overline{0}$

$\Rightarrow x \in I$ and $y+x-y \notin I$

$(x(y+x)-x y) \notin I$.

This is a contradiction. 


\section{V.Vetrivel and P.Murugadas}

Therefore, $y+x-y \in I$

$\Rightarrow \bar{\lambda}_{I}(y+x-y)=\overline{1}$

$\bar{\lambda}_{I}(x)=\bar{\lambda}_{I}(y+x-y)$.

Suppose, $\bar{\lambda}_{I}(x y) \leq \bar{\lambda}_{I}(x) \bar{\lambda}_{I}(x)=\overline{1} \Rightarrow x \in I, \quad \bar{\lambda}_{I}(x y)=\overline{0}$ and $x y \notin I$.

This is a contradiction.

$\bar{\lambda}_{I}(x y)=\overline{1}$.

Therefore, $\bar{\lambda}_{I}(x y) \geq \bar{\lambda}_{I}(x)$.

Definition 3.2. [1] An i-v fuzzy subgroup $\bar{\lambda}$ of $N$ is called an i-v fuzzy bi-ideal of $N$, if $\left(\bar{\lambda} \circ \bar{\lambda}_{N} \circ \bar{\lambda}\right) \cap\left(\bar{\lambda}_{\bar{\lambda}} \circ \bar{\lambda}_{N} * \bar{\lambda}\right) \leq \bar{\lambda}$.

Definition 3.3. [1] An i-v fuzzy set $\bar{\lambda}$ of a near-ring $N$ is called an i-v fuzzy bi-ideal of $N$, if

(1) $\bar{\lambda}(x-y) \geq \min ^{i}\{\bar{\lambda}(x), \bar{\lambda}(y)\}$ for all $x, y, z \in N$.

(2) $\bar{\lambda}(x y z) \geq \min ^{i}\{\bar{\lambda}(x), \bar{\lambda}(z)\}$ for all $x, y, z \in N$.

Lemma 3.4. For any non-empty subsets $X$ and $Y$ of near-ring $N$, we have

(1) $\bar{\lambda}_{X} \circ \bar{\lambda}_{Y}=\bar{\lambda}_{X Y}$

(2) $\bar{\lambda}_{X} \cap \bar{\lambda}_{Y}=\bar{\lambda}_{X \cap Y}$

(3) $\bar{\lambda}_{X} * \bar{\lambda}_{Y}=\bar{\lambda}_{X * Y}$

\section{Proof:}

(1) Let $x \in N$. Suppose $x \in X Y$. Then $x=a b$ for some $a \in X$ and $b \in Y$. Thus we have,

$$
\begin{gathered}
\left(\bar{\lambda}_{X} \circ \bar{\lambda}_{Y}\right)(x)=\sup _{x=a b}\left\{\min ^{i}\left\{\bar{\lambda}_{X}(a), \bar{\lambda}_{Y}(b)\right\}\right\} \\
\geq \min ^{i}\left\{\bar{\lambda}_{X}(a), \bar{\lambda}_{Y}(b)\right\} \\
=\min ^{i}\{\overline{1}, \overline{1}\} \\
=\overline{1} \\
\bar{\lambda}_{X Y}=\overline{1} . \\
\left(\bar{\lambda}_{X} \circ \bar{\lambda}_{Y}\right)=\bar{\lambda}_{X Y} .
\end{gathered}
$$

In this case $x \notin X Y$, we have $x \neq a b$ for any $a \in X$ and $b \in Y$.

If $x=u v$ for some $u, v \in N$, then we have,

$$
\begin{aligned}
& \left(\bar{\lambda}_{X} \circ \bar{\lambda}_{Y}\right)(x)=\sup _{x=u v}^{i}\left\{\min ^{i}\left\{\bar{\lambda}_{X}(u), \bar{\lambda}_{Y}(v)\right\}\right\} \\
& =\sup _{x=u v}^{i}\left\{\min ^{i}\{\overline{0}, \overline{0}\}\right\} \\
& =\overline{0}
\end{aligned}
$$


Interval Valued Fuzzy Prime Bi-Ideals and Interval Valued Fuzzy Strongly Prime BiIdeals in Near-Rings

$\bar{\lambda}_{X Y}=\overline{0}$.

In all the cases, we have

$\left(\bar{\lambda}_{X} \circ \bar{\lambda}_{Y}\right)(x)=\bar{\lambda}_{X Y}(x)$ for all $x \in N$.

Thus, $\bar{\lambda}_{X} \circ \bar{\lambda}_{Y}=\bar{\lambda}_{X Y}$.

(2) Let $a \in N$. Suppose $a \in X \cap Y$. Then $a \in X$ and $a \in Y$, Thus we have,

$\left(\bar{\lambda}_{X} \cap \bar{\lambda}_{Y}\right)(a)=\min ^{i}\left\{\bar{\lambda}_{X}(a), \bar{\lambda}_{Y}(a)\right\}=\min ^{i}\{\overline{1}, \overline{1}\}=\overline{1}$

$\bar{\lambda}_{X \cap Y}=\overline{1}$.

Suppose $a \notin X \cap Y$. Then $a \notin X$ and $a \notin Y$.

Hence,

$$
\begin{aligned}
& \left(\bar{\lambda}_{X} \cap \bar{\lambda}_{Y}\right)(a)=\min ^{i}\left\{\bar{\lambda}_{X}(a), \bar{\lambda}_{Y}(a)\right\}=\min ^{i}\{\overline{0}, \overline{0}\}=\overline{0} \\
& \bar{\lambda}_{X \cap Y}=\overline{0} .
\end{aligned}
$$

Thus we get $\bar{\lambda}_{X} \cap \bar{\lambda}_{Y}=\bar{\lambda}_{X \cap Y}$.

(3) $\bar{\lambda}_{X} * \bar{\lambda}_{Y}=\bar{\lambda}_{X * Y}$.

Let $x \in N$, Suppose $x \in X * Y$. Then $x=a(b+c)-a b ; a \in X$ and $b \in Y, c \in Y$

$$
\left(\bar{\lambda}_{X} * \bar{\lambda}_{Y}\right)(x)=\sup _{x=a(b+c)-a b}^{i}\left\{\min ^{i}\left\{\bar{\lambda}_{X}(a), \bar{\lambda}_{Y}(c)\right\}\right\}=\sup _{x=a(b+c)-a b}^{i}\{\overline{1}, \overline{1}\}=\min ^{i}\{\overline{1}, \overline{1}\}=\overline{1} \text {, and }
$$

$\bar{\lambda}_{X * Y}=\overline{1}$.

In the case $x \notin X * Y$, If $x \neq a(b+c)-a b$ for some $x \in N$. Then we have, $\bar{\lambda}_{X * Y}=\overline{0}$. similarly,

$\bar{\lambda}_{X} * \bar{\lambda}_{Y}=\overline{0}$

Lemma 3.5. Let $I$ be a subgroup of $(N,+), I$ is a bi-ideal of $N$ if and only if $\bar{\lambda}_{I}$ is an i-v fuzzy bi-ideal of $N$.

Proof: Assume that $I$ is a bi-ideal of $N$.

$$
\left(\bar{\lambda}_{I} \circ \bar{\lambda}_{N} \circ \bar{\lambda}_{I}\right) \cap\left(\bar{\lambda}_{I} \circ \bar{\lambda}_{N} * \bar{\lambda}_{I}\right) \leq \bar{\lambda}_{I}
$$

$\operatorname{Now}\left(\bar{\lambda}_{I} \circ \bar{\lambda}_{N} \circ \bar{\lambda}_{I}\right) \cap\left(\bar{\lambda}_{I} \circ \bar{\lambda}_{N} * \bar{\lambda}_{I}\right) \subseteq \bar{\lambda}_{I N I} \cap \bar{\lambda}_{I N * I}$

$\subseteq \bar{\lambda}_{I \cap \bar{\lambda}_{l^{*} I}}($ Since $I$ is a bi-ideal of $N)$

$=\bar{\lambda}_{I} \cap \bar{\lambda}_{I}=\bar{\lambda}_{I}$.

Therefore, $\bar{\lambda}_{I}$ is an i-v fuzzy bi-ideal of $N$.

Lemma 3.6. Let $\bar{\lambda}$ and $\bar{\mu}$ be two i-v fuzzy bi-ideal of a near-ring $N$. Then $\bar{\lambda} \wedge \bar{\mu}$ is an i-v fuzzy bi-ideal of $N$.

Proof: Let $\bar{\lambda}$ and $\bar{\mu}$ be a i-v fuzzy bi-ideals of $N$. 
V.Vetrivel and P.Murugadas

$\left((\bar{\lambda} \wedge \bar{\mu}) \circ \bar{\lambda}_{N} \circ(\bar{\lambda} \wedge \bar{\mu})\right) \cap\left((\bar{\lambda} \wedge \bar{\mu}) \circ \bar{\lambda}_{N} *(\bar{\lambda} \wedge \bar{\mu})\right) \leq\left(\bar{\lambda} \circ \bar{\lambda}_{N} \circ \bar{\lambda}\right) \cap\left(\bar{\lambda} \circ \bar{\lambda}_{N} * \bar{\lambda}\right) \leq \bar{\lambda}$

Similarly,

$\left((\bar{\lambda} \wedge \bar{\mu}) \circ \bar{\lambda}_{N} \circ(\bar{\lambda} \wedge \bar{\mu})\right) \cap\left((\bar{\lambda} \wedge \bar{\mu}) \circ \bar{\lambda}_{N} *(\bar{\lambda} \wedge \bar{\mu})\right) \leq\left(\bar{\mu} \circ \bar{\lambda}_{N} \circ \bar{\mu}\right) \cap\left(\bar{\mu} \circ \bar{\lambda}_{N} * \bar{\mu}\right) \leq \bar{\mu}$

Therefore, $\left((\bar{\lambda} \wedge \bar{\mu}) \circ \bar{\lambda}_{N} \circ(\bar{\lambda} \wedge \bar{\mu})\right) \cap\left((\bar{\lambda} \wedge \bar{\mu}) \circ \bar{\lambda}_{N} *(\bar{\lambda} \wedge \bar{\mu})\right) \leq \bar{\lambda} \wedge \bar{\mu}$.

Thus $\bar{\lambda} \wedge \bar{\mu}$. is an i-v fuzzy bi-ideal of $N$.

\section{Prime, strongly prime and semi-prime I-V fuzzy bi-ideals}

Definition 4.1. An i-v fuzzy bi-ideal $\bar{\lambda}$ of a near-ring $N$ is called a prime i-v fuzzy biideal of $N$, if for any i-v fuzzy bi-ideals $\bar{\mu}, \bar{v}$ of $N, \bar{\mu} \circ \bar{v} \leq \bar{\lambda}$ implies $\bar{\mu} \leq \bar{\lambda}$ or $\bar{v} \leq \bar{\lambda}$.

Definition 4.2. An i-v fuzzy bi-ideal $\bar{\lambda}$ of a near-ring $N$ is called a strongly prime fuzzy bi-ideal of $N$, if for any i-v fuzzy bi-ideals $\bar{\mu}, \bar{v}$ of $N,(\bar{\mu} \circ \bar{v}) \wedge(\bar{v} \circ \bar{\mu}) \leq \bar{\lambda}$ implies $\bar{\mu} \leq \bar{\lambda}$ or $\bar{v} \leq \bar{\lambda}$.

Definition 4.2. An i-v fuzzy bi-ideal $\bar{\mu}$ of a near-ring $N$ is said to be idempotent if $\bar{\mu}=\bar{\mu} \circ \bar{\mu}=\bar{\mu}^{2}$.

Definition 4.3. An i-v fuzzy bi-ideal $\bar{\lambda}$ of a near-ring $N$ is said to be a semi-prime i-v fuzzy bi-ideal of $N$, if $\bar{\mu} \circ \bar{\mu}=\bar{\mu}^{2} \leq \bar{\lambda}$ implies $\bar{\mu} \leq \bar{\lambda}$ for every i-v fuzzy bi-ideal $\bar{\mu}$ of $N$.

Proposition 4.4. Every strongly prime i-v fuzzy bi-ideal of a near-ring $N$ is a prime i-v fuzzy bi-ideal of $N$.

Proof: Let $\bar{\lambda}$ be a strongly prime i-v fuzzy bi-ideal of a near-ring $N$. Now let $\bar{\mu}, \bar{v}$ be two i-v fuzzy bi-ideals of $N$ such that $\bar{\mu} \circ \bar{v} \leq \bar{\lambda}$. Then $(\bar{\mu} \circ \bar{v}) \wedge(\bar{v} \circ \bar{\mu}) \leq \bar{\lambda}$. Thus by hypothesis, $\bar{\mu} \leq \bar{\lambda}$ or $\bar{v} \leq \bar{\lambda}$. Hence $\bar{\lambda}$ is a prime i-v fuzzy bi-ideal of $N$.

Proposition 4.5. Every prime i-v fuzzy bi-ideal of a near-ring $N$ is a semi-prime i-v fuzzy bi-ideal of $N$.

Proof: Let $\bar{\lambda}$ be a prime i-v fuzzy bi-ideal of a near-ring $N$. Now let $\bar{\mu}$ be any i-v fuzzy bi-ideal of $N$ such that $\bar{\mu} \circ \bar{\mu} \leq \bar{\lambda}$. Then by hypothesis $\bar{\mu} \leq \bar{\lambda}$. Hence $\bar{\lambda}$ is a semi-prime i-v fuzzy bi-ideal of $N$.

Remarks 4.6. Every i-v fuzzy prime bi-ideal of $N$ is semi-prime. But every i-v fuzzy semi-prime bi-ideal of $N$ is not prime.

Consider the i-v fuzzy bi-ideals $\bar{\lambda}, \bar{\mu}$ and $\bar{v}$ of $N$ given by

Let $N=\{0,1,2\}$ be a set with two binary operations as follows, 
Interval Valued Fuzzy Prime Bi-Ideals and Interval Valued Fuzzy Strongly Prime BiIdeals in Near-Rings

\begin{tabular}{|l|l|l|l|}
\hline+ & 0 & 1 & 2 \\
\hline 0 & 0 & 1 & 2 \\
\hline 1 & 1 & 0 & 1 \\
\hline 2 & 2 & 1 & 0 \\
\hline
\end{tabular}

\begin{tabular}{|l|l|l|l|}
\hline$\cdot$ & 0 & 1 & 2 \\
\hline 0 & 0 & 0 & 0 \\
\hline 1 & 0 & 1 & 1 \\
\hline 2 & 0 & 1 & 2 \\
\hline
\end{tabular}

$$
\begin{array}{lrrrl}
(\bar{\lambda})(0) & =[0.7,0.8] & (\bar{\lambda})(1)=[0.6,0.65] & (\bar{\lambda})(2)=[0.4,0.5] \\
(\bar{\mu})(0)=[1,1] & (\bar{\lambda})(0)=[0.7,0.75] & (\bar{\lambda})(0)=[0.7,0.75] \\
(\bar{v})(0)=[0.7,0.75] & (\bar{v})(1)=[0.64,0.65] & (\bar{v})(0)=[0.3,0.37]
\end{array}
$$

Clearly, $\bar{\lambda}$ is an i-v fuzzy semi-prime bi-ideal. Then $(\bar{\mu} \circ \bar{v})(0)=[0.7,0.75] \quad(\bar{\mu} \circ \bar{v})(1)=[0.5,0.5](\bar{\mu} \circ \bar{v})(2)=[0.3,0.35]$ $\bar{\mu} \circ \bar{\nu} \leq \bar{\lambda}$ but neither $\bar{\mu} \leq \bar{\lambda}$ nor $\bar{v} \leq \bar{\lambda}$. Hence $\bar{\lambda}$ is not a prime i-v fuzzy bi-ideal of $N$.

Lemma 4.7. Product of two $\mathrm{i}-\mathrm{v}$ fuzzy bi-ideals of $N$ is an i-v fuzzy bi-ideal of $N$.

Proof: Let $\bar{\lambda}$ and $\bar{\mu}$ be two i-v fuzzy bi-ideals of $N$. We have to show that $(\bar{\lambda} \circ \bar{\mu})$ is an i-v fuzzy bi-ideal of $N$. Let $(\bar{\lambda} \circ \bar{\mu})(x-y)=\bar{\lambda}(\bar{\mu}(x-y)) \geq \bar{\lambda}\left\{\min ^{i}(\bar{\mu}(x), \bar{\mu}(y))\right\}$ (as $\bar{\mu}$ is a i-v fuzzy bi-ideal of $N$ ).

$$
\begin{aligned}
& =\bar{\lambda}\left\{\min ^{i}(\bar{\mu}(x))\right\}, \bar{\lambda}\left\{\min ^{i}(\bar{\mu}(y))\right\} \\
& =\min ^{i}\{(\bar{\lambda} \circ \bar{\mu})(x),(\bar{\lambda} \circ \bar{\mu})(y)\}
\end{aligned}
$$

Therefore, $(\bar{\lambda} \circ \bar{\mu})(x-y) \geq \min ^{i}\{(\bar{\lambda} \circ \bar{\mu})(x),(\bar{\lambda} \circ \bar{\mu})(y)\}$.

(ii) $(\bar{\lambda} \circ \bar{\mu}) \circ \bar{\lambda}_{N} \circ(\bar{\lambda} \circ \bar{\mu}) \cap(\bar{\lambda} \circ \bar{\mu}) \circ \bar{\lambda}_{N} *(\bar{\lambda} \circ \bar{\mu})=$ $\left[(\bar{\lambda} \circ \bar{\mu}) \circ \bar{\lambda}_{N} \circ \bar{\lambda} \cap(\bar{\lambda} \circ \bar{\mu}) \circ \bar{\lambda}_{N} * \bar{\lambda}\right] \bar{\mu}$ $\left.\leq\left[\left(\bar{\lambda} \circ \bar{\lambda}_{N} \circ \bar{\lambda}_{N} \circ \bar{\lambda}\right) \cap(\bar{\lambda} \circ \bar{\mu}) \circ \bar{\lambda}_{N} * \bar{\lambda}\right)\right] \bar{\mu} \leq\left[\left(\bar{\lambda}_{0} \circ \bar{\lambda}_{N} \circ \bar{\lambda}\right) \cap\left(\bar{\lambda}_{\lambda} \circ \bar{\lambda}_{N} \circ \bar{\lambda}_{N} * \bar{\lambda}\right)\right] \bar{\mu} \leq \bar{\lambda} \circ \bar{\mu}$ Hence $\bar{\lambda} \circ \bar{\mu}$ is a i-v fuzzy bi-ideal of near-ring $N$.

\section{Irreducible and strongly irreducible I-V fuzzy bi-ideals}

Definition 5.1. An i-v fuzzy bi-ideal $\bar{\lambda}$ of near-ring $N$ is said to be an irreducible i-v fuzzy bi-ideal of $N$, if for any i-v fuzzy bi-ideals $\bar{\mu}$ and $\bar{v}$ of $N, \bar{\mu} \wedge \bar{v}=\bar{\lambda}$ implies $\bar{\mu}=\bar{\lambda}$ or $\bar{v}=\bar{\lambda}$.

Definition 5.2. An i-v fuzzy bi-ideal $\bar{\lambda}$ of near-ring $N$ is said to be a strongly irreducible i-v fuzzy bi-ideal of $N$, if for any i-v fuzzy bi-ideals $\bar{\mu}$ and $\bar{v}$ of $N$, $\bar{\mu} \wedge \bar{v} \leq \bar{\lambda}$ implies $\bar{\mu} \leq \bar{\lambda}$ or $\bar{v} \leq \bar{\lambda}$. 


\section{V.Vetrivel and P.Murugadas}

Proposition 5.3. Every strongly irreducible semi-prime i-v fuzzy bi-ideal of a near-ring $N$ is a strongly prime i-v fuzzy bi-ideal of $N$.

Proof: Let $\bar{\lambda}$ be a strongly irreducible semi-prime i-v fuzzy bi-ideal of a near-ring $N$. Let $\bar{\mu}, \bar{v}$ be any i-v fuzzy bi-ideals of $N$ such that $(\bar{\mu} \circ \bar{v}) \wedge(\bar{v} \circ \bar{\mu}) \leq \bar{\lambda}$. As $(\bar{\mu} \wedge \bar{v}) \circ(\bar{\mu} \wedge \bar{v})=(\bar{\mu} \wedge \bar{v})^{2} \leq(\bar{\mu} \circ \bar{v}) \quad$ and $\quad(\bar{\mu} \wedge \bar{v}) \circ(\bar{\mu} \wedge \bar{v})=(\bar{\mu} \wedge \bar{v})^{2} \leq(\bar{v} \circ \bar{\mu})$, implies $(\bar{\mu} \wedge \bar{v})^{2} \leq(\bar{\mu} \circ \bar{v}) \wedge(\bar{v} \circ \bar{\mu}) \leq \bar{\lambda}$. So $\bar{\mu} \wedge \bar{v} \leq \bar{\lambda}$, as $\bar{\lambda}$ is a semi-prime i-v fuzzy bi-ideal of $N$. Thus either $\bar{\mu} \leq \bar{\lambda}$ or $\bar{v} \leq \bar{\lambda}$, because $\bar{\lambda}$ is a strongly irreducible $\mathrm{i}-$ $\mathrm{v}$ fuzzy bi-ideal of $N$. Hence $\bar{\lambda}$ is a strongly prime i-v fuzzy bi-ideal of $N$.

Theorem 5.4. Let $\bar{\lambda}$ be an i-v fuzzy bi-ideal of a near-ring $N$ with $\bar{\lambda}(a)=\alpha$ where $a \in N$ and $\alpha \in[0,1]$. Then there exists an irreducible i-v fuzzy bi-ideal $\bar{\mu}$ of $N$ such that $\bar{\lambda} \leq \bar{\mu}$ and $\bar{\mu}(a)=\alpha$.

Proof: Let $X=\{\bar{v}: \bar{v}$ is an i-v fuzzy bi-ideal of $N, \bar{v}(a)=\alpha$ and $\bar{\lambda} \leq \bar{v}\}$, then $X \neq \phi$ (non-empty), as $\bar{\lambda} \in X$. The collection $X$ is a partially ordered under inclusion. If $Y=\left\{\overline{v_{i}}: \overline{v_{i}}\right.$ is an i-v fuzzy bi-ideal of $\mathrm{N}, \overline{v_{i}}(a)=\alpha$ and $\bar{\lambda} \leq \overline{v_{i}}$ for all $\left.i \in I\right\}$ is any totally ordered subset of $X$, then $\vee_{i \in I} \bar{v}_{i}$ is an i-v fuzzy bi-ideal of $N$ such that $\bar{\lambda} \leq \vee_{i \in I} \bar{v}_{i}$. Indeed, if $a, b, x \in N$, then

$\left(\vee_{i \in I} \overline{v_{i}}\right)(a-b)=\vee_{i \in I}\left(\bar{v}_{i}(a-b)\right)$

$\geq \vee_{i \in I}\left(\overline{v_{i}}(a) \wedge \bar{v}_{i}(b)\right)$ (as each $\overline{v_{i}}$ is an i-v fuzzy bi-ideal of $\left.N\right)$.

$=\left(\vee_{i \in I} \overline{V_{i}}(a)\right) \wedge\left(\vee_{i \in I} \overline{v_{i}}(b)\right)$

$=\left(\vee_{i \in I} \bar{v}_{i}\right)(a) \wedge\left(\vee_{i \in I} \overline{\nu_{i}}\right)(b)$

and $\left(\vee_{i \in I} \bar{v}_{i}\right)(a b)=\vee_{i \in I}\left(\bar{v}_{i}(a b)\right)$

$\geq \vee_{i \in I}\left(\overline{v_{i}}(a) \wedge \overline{v_{i}}(b)\right)$ (as each $\overline{v_{i}}$ is an i-v fuzzy bi-ideal of $N$ ).

$=\left(\vee_{i \in I} \overline{V_{i}}(a)\right) \wedge\left(\vee_{i \in I} \overline{V_{i}}(b)\right)$

$=\left(\vee_{i \in I} \overline{V_{i}}\right)(a) \wedge\left(\vee_{i \in I} \overline{v_{i}}\right)(b)$

Now $\left(\vee_{i \in I} \overline{v_{i}}\right)(a x b)=\vee_{i \in I}\left(\overline{v_{i}}(a x b)\right)$

$\geq \vee_{i \in I}\left(\overline{v_{i}}(a) \wedge \overline{v_{i}}(b)\right)$ ( as each $\overline{v_{i}}$ is an i-v fuzzy bi-ideal of $N$ ).

$=\left(\vee_{i \in I} \overline{v_{i}}(a)\right) \wedge\left(\vee_{i \in I} \overline{v_{i}}(b)\right)$

$=\left(\vee_{i \in I} \overline{\nu_{i}}\right)(a) \wedge\left(\vee_{i \in I} \overline{\nu_{i}}\right)(b)$

Hence $\vee_{i \in I} \overline{v_{i}}$ is an i-v fuzzy bi-ideal of $N$. As $\bar{\lambda} \leq \overline{v_{i}}$ for all $i \in I$. This implies $\bar{\lambda} \leq \vee_{i \in I} \overline{v_{i}}$. Also

$=\left(\vee_{i \in I} \overline{v_{i}}\right)(a)=\vee_{i \in I} \overline{v_{i}}(a)=\alpha$. 
Interval Valued Fuzzy Prime Bi-Ideals and Interval Valued Fuzzy Strongly Prime BiIdeals in Near-Rings

Thus $\vee_{i \in I} \overline{\bar{v}_{i}} \in X$ and $\vee_{i \in I} \overline{v_{i}}$ is an upper bound of $Y$. Hence by Zorn's lemma, there exists an i-v fuzzy bi-ideal $\bar{\mu}$ of $N$ which is maximal with the properly $\bar{\lambda} \leq \bar{\mu}$ and $\bar{\mu}(a)=\alpha$. Now we show that $\bar{\mu}$ is an irreducible i-v fuzzy bi-ideal of $N$. For this, suppose that for any i-v fuzzy bi-ideals $\bar{\mu}_{1}, \bar{\mu}_{2}$ of $N$, we have $\bar{\mu}_{1} \wedge \bar{\mu}_{2}=\bar{\mu}$. This implies $\bar{\mu} \leq \bar{\mu}_{1}$ and $\bar{\mu} \leq \bar{\mu}_{2}$. We claim that $\bar{\mu}=\bar{\mu}_{1}$ or $\bar{\mu}=\bar{\mu}_{2}$. On contrary, suppose that $\bar{\mu} \neq \bar{\mu}_{1}$ and $\bar{\mu} \neq \bar{\mu}_{2}$. This implies $\bar{\mu}<\bar{\mu}_{1}$ and $\bar{\mu}<\bar{\mu}_{2}$. So $\bar{\mu}_{1}(a) \neq \alpha$ and $\bar{\mu}_{2}(a) \neq \alpha$, as $\bar{\mu}(a)=\alpha$. Hence $\left(\bar{\mu}_{1} \wedge \bar{\mu}_{2}\right)(a)=\bar{\mu}_{1}(a) \wedge \bar{\mu}_{2}(a) \neq \alpha$. Which is a contradiction to the fact that $\bar{\mu}_{1}(a) \wedge \bar{\mu}_{2}(a)=\bar{\mu}(a)=\alpha$. Hence either $\bar{\mu}=\bar{\mu}_{1}$ or $\bar{\mu}=\bar{\mu}_{2}$. Thus $\bar{\mu}$ is an irreducible i-v fuzzy bi-ideal of $N$.

Theorem 5.5. For a near-ring $N$, the following assertions are equivalent:

(1) $\bar{\lambda} \circ \bar{\lambda}=\bar{\lambda}$ for every i-v fuzzy bi-ideal of $N$.

(2) $\bar{\mu} \wedge \bar{v}=(\bar{\mu} \circ \bar{v}) \wedge(\bar{v} \circ \bar{\mu})$ for all i-v fuzzy bi-ideals $\bar{\mu}$ and $\bar{v}$ of $N$.

(3) Each i-v fuzzy bi-ideal of $N$ is i-v fuzzy semi-prime.

(4) Each proper i-v fuzzy bi-ideal of $N$ is the intersection of irreducible semi-prime i-v fuzzy bi-ideals of $N$ which contain it.

\section{Proof:}

(1) $\Rightarrow$ (2): Let $\bar{\mu}$ and $\bar{v}$ be two i-v fuzzy bi-ideals of $N$. Then by Lemma 3.6, $\bar{\mu} \wedge \bar{v}$ is also an i-v fuzzy bi-ideal of $N$. Thus by hypothesis, we have $\bar{\mu} \wedge \bar{v}=(\bar{\mu} \wedge \bar{v}) \circ(\bar{\mu} \wedge \bar{v}) \leq \bar{\mu} \circ \bar{v} . \quad$ Similarly $\quad \bar{\mu} \wedge \bar{v} \leq \bar{v} \circ \bar{\mu} . \quad$ Implies $\bar{\mu} \wedge \bar{v} \leq(\bar{\mu} \circ \bar{v}) \wedge(\bar{v} \circ \bar{\mu})$. Now $\bar{\mu} \circ \bar{v}$ and $\bar{v} \circ \bar{\mu}$, being products of two i-v fuzzy biideals of $N$, are i-v fuzzy bi-ideals of $N$. Also $(\bar{\mu} \circ \bar{v}) \wedge(\bar{v} \circ \bar{\mu})$ is an i-v fuzzy bi-ideal of $N$, by Lemma 3.6. Thus by hypothesis, we have

$(\bar{\mu} \circ \bar{v}) \wedge(\bar{v} \circ \bar{\mu})=((\bar{\mu} \circ \bar{v}) \wedge(\bar{v} \circ \bar{\mu})) \circ((\bar{\mu} \circ \bar{v}) \wedge(\bar{v} \circ \bar{\mu})) \leq(\bar{\mu} \circ \bar{v}) \circ(\bar{v} \circ \bar{\mu})=\bar{\mu} \circ \bar{v} \circ \bar{\mu}$ as $\bar{v} \circ \bar{v}=\bar{V}$ (by hypothesis)

$\leq \bar{\mu} \circ \bar{\lambda}_{N} \circ \bar{\mu}$ as $\bar{v} \leq \bar{\lambda}_{N}$

$\leq \bar{\mu} \quad($ as $\bar{\mu}$ is an i-v fuzzy bi-ideal of $N)$.

Similarly $(\bar{\mu} \circ \bar{v}) \wedge(\bar{v} \circ \bar{\mu}) \leq \bar{v}$. Thus $(\bar{\mu} \circ \bar{v}) \wedge(\bar{v} \circ \bar{\mu}) \leq \bar{\mu} \wedge \bar{v}$.

Hence $(\bar{\mu} \circ \bar{v}) \wedge(\bar{v} \circ \bar{\mu})=\bar{\mu} \wedge \bar{v}$

(2) $\Rightarrow$ (3): Let $\bar{\mu}$ be a i-v fuzzy bi-ideal of $N$ such that $\bar{\lambda}^{2} \leq \bar{\mu}$ for any bi-ideal of $\bar{\lambda}$ of $N$. Then by hypothesis, $\bar{\lambda}=\bar{\lambda} \wedge \bar{\lambda}=(\bar{\lambda} \circ \bar{\lambda}) \wedge(\bar{\lambda} \circ \bar{\lambda})=\bar{\lambda} \circ \bar{\lambda}=\bar{\lambda}^{2} \leq \bar{\mu}$

This implies $\bar{\lambda} \leq \bar{\mu}$. Thus $\bar{\lambda}$ is semi-prime i-v fuzzy bi-ideal of $N$. Hence every i-v fuzzy bi-ideal of $N$ is semi-prime.

(3) $\Rightarrow$ (4): Let $\bar{\lambda}$ be a proper i-v fuzzy bi-ideal of $N$ and $\left\{\bar{\lambda}_{i}: i \in I\right\}$ be the collection of all irreducible i-v fuzzy bi-ideals of $N$ such that $\bar{\lambda} \leq \bar{\lambda}_{i}$ for all $i \in I$. This implies $\bar{\lambda} \leq \wedge_{i \in I} \bar{\lambda}_{i}$. Let $a \in N$ then by Theorem 5.4, there exists an irreducible i-v fuzzy bi- 


\section{V.Vetrivel and P.Murugadas}

ideal $\bar{\lambda}_{\alpha}$ of $N$ such that $\bar{\lambda} \leq \bar{\lambda}_{\alpha}$ and $\bar{\lambda}(a)=\bar{\lambda}_{\alpha}(a)$. This implies $\bar{\lambda}_{\alpha} \in\left\{\bar{\lambda}_{i}: i \in I\right\}$. Thus $\wedge_{i \in I} \bar{\lambda}_{i} \leq \bar{\lambda}_{\alpha}$. So $\wedge_{i \in I} \bar{\lambda}_{i}(a) \leq \bar{\lambda}_{\alpha}(a)=\bar{\lambda}(a)$ for all $a \in N$. This implies $\wedge_{i \in I} \bar{\lambda}_{i} \leq \bar{\lambda}$. Hence $\wedge_{i \in I} \bar{\lambda}_{i}=\bar{\lambda}$. By hypothesis, each i-v fuzzy bi-ideal of $N$ is semiprime. Thus each i-v fuzzy bi-ideal of $N$ is the intersection of all irreducible semi-prime i-v fuzzy bi-ideals of $N$ which contain it.

(4) $\Rightarrow(1)$ : Let $\bar{\lambda}$ be an i-v fuzzy bi-ideal of $N$. Then by the definition of i-v fuzzy biideal we have, $\bar{\lambda}^{2}=\bar{\lambda} \circ \bar{\lambda} \leq \bar{\lambda}$. Also $\bar{\lambda}^{2}=\bar{\lambda} \circ \bar{\lambda}$, being the product of two i-v fuzzy biideals of $N$ is an i-v fuzzy bi-ideal of $N$. Then by hypothesis, $\bar{\lambda}^{2}=\wedge_{i \in I} \bar{\lambda}_{i}$, where each $\bar{\lambda}_{i}$ is an irreducible semi-prime i-v fuzzy bi-ideal of $N$ such that $\bar{\lambda}^{2} \leq \bar{\lambda}_{i}$ for all $i \in I$. This implies $\bar{\lambda} \leq \bar{\lambda}_{i}$ for all $i \in I$ because each $\bar{\lambda}_{i}$ is a semi-prime i-v fuzzy bi-ideal of $N$ Thus $\bar{\lambda} \leq \wedge_{i \in I} \bar{\lambda}_{i}=\bar{\lambda}^{2}$. Hence $\bar{\lambda}^{2}=\bar{\lambda}$,

Therefore, $\bar{\lambda} \circ \bar{\lambda}=\bar{\lambda}$.

Proposition 5.6. Let each i-v fuzzy bi-ideal of a near-ring $N$ is idempotent. Then the following assertions for a i-v fuzzy bi-ideal of $N$ are equivalent:

(1) $\bar{\lambda}$ is strongly irreducible.

(2) $\bar{\lambda}$ is strongly prime.

Proof:

$(1) \Rightarrow(2)$ : Let each i-v fuzzy bi-ideal of a near-ring $N$ is idempotent and $\bar{\lambda}$ be a strongly irreducible i-v fuzzy bi-ideal of $N$. Suppose that $\bar{\mu}$ and $\bar{v}$ be two i-v fuzzy biideals of $N$ such that $(\bar{\mu} \circ \bar{v}) \wedge(\bar{v} \circ \bar{\mu}) \leq \bar{\lambda}$. By Theorem, $\bar{\mu} \wedge \bar{v}=\bar{\mu} \circ \bar{v} \wedge \bar{v} \circ \bar{\mu} \leq \bar{\lambda}$. Implies either $\bar{\mu} \leq \bar{\lambda}$ or $\bar{v} \leq \bar{\lambda}$, as $\bar{\lambda}$ is strongly irreducible. So $\bar{\lambda}$ is strongly prime $\mathrm{i}-$ v fuzzy bi-ideal of $N$.

(2) $\Rightarrow(1)$ : Suppose $\bar{\lambda}$ is a strongly prime i-v fuzzy bi-ideal of $N$. Let $\bar{\mu}$ and $\bar{v}$ be any i-v fuzzy bi-ideals of $N$ such that $\bar{\mu} \wedge \bar{v} \leq \bar{\lambda}$. By Theorem 5.5 $(\bar{\mu} \circ \bar{v}) \wedge(\bar{v} \circ \bar{\mu})=\bar{\mu} \wedge \bar{v} \leq \bar{\lambda}$, so $(\bar{\mu} \circ \bar{v}) \wedge(\bar{v} \circ \bar{\mu}) \leq \bar{\lambda}$. Implies either $\bar{\mu} \leq \bar{\lambda}$ or $\bar{v} \leq \bar{\lambda}$, as $\bar{\lambda}$ is a strongly prime i-v fuzzy bi-ideal of $N$. Thus $\bar{\lambda}$ is strongly irreducible.

Theorem 5.7 Each i-v fuzzy bi-ideal of a near-ring $N$ is strongly prime if and only if each i-v fuzzy bi-ideal of $N$ is idempotent and the set of i-v fuzzy bi-ideals of $N$ is totally ordered by inclusion.

Proof: Suppose that each i-v fuzzy bi-ideal of near-ring $N$ is strongly prime, then each iv fuzzy bi-ideal of $N$ is semi-prime. Thus by Theorem 5.5 each i-v fuzzy bi-ideal of $N$ is idempotent. Now we show that the set of i-v fuzzy bi-ideals of $N$ is totally ordered by inclusion. For this let $\bar{\mu}$ and $\bar{v}$ be any two i-v fuzzy bi-ideals of $N$. Then by Theorem 5.5, $(\bar{\mu} \circ \bar{v}) \wedge(\bar{v} \circ \bar{\mu})=\bar{\mu} \wedge \bar{v}$, implies $(\bar{\mu} \circ \bar{v}) \wedge(\bar{v} \circ \bar{\mu}) \leq \bar{\mu} \wedge \bar{v}$. As each i-v fuzzy bi-ideal of $N$ is strongly prime, so is $\bar{\mu} \wedge \bar{v}$. Thus either $\bar{\mu} \leq \bar{\mu} \wedge \bar{v}$ or $\bar{v} \leq \bar{\mu} \wedge \bar{v}$. If 
Interval Valued Fuzzy Prime Bi-Ideals and Interval Valued Fuzzy Strongly Prime BiIdeals in Near-Rings

$\bar{\mu} \leq \bar{\mu} \wedge \bar{v}$, implies $\bar{\mu} \leq \bar{v}$ and if $\bar{v} \leq \bar{\mu} \wedge \bar{v}$, implies $\bar{v} \leq \bar{\mu}$. So the set of i-v fuzzy biideals of $N$ is totally ordered by inclusion.

Conversely, assume that each i-v fuzzy bi-ideal of $N$ is idempotent and the set of i-v fuzzy bi-ideals of $N$ is totally ordered by inclusion. Let $\bar{\lambda}$ be an arbitrary i-v fuzzy biideal of $N$ and $\bar{\mu}, \bar{v}$ be any i-v fuzzy bi-ideals of $N$ such that $(\bar{\mu} \circ \bar{v}) \wedge(\bar{v} \circ \bar{\mu}) \leq \bar{\lambda}$.

By Theorem 5.5, $\bar{\mu} \wedge \bar{v}=(\bar{\mu} \circ \bar{v}) \wedge(\bar{v} \circ \bar{\mu}) \leq \bar{\lambda}$ implies $\bar{\mu} \wedge \bar{v} \leq \bar{\lambda} \ldots(i)$

Since the set of i-v fuzzy bi-ideals of $N$ is totally ordered by inclusion. So either $\bar{\mu} \leq \bar{v}$ or $\bar{v} \leq \bar{\mu}$, implies either $\bar{\mu} \wedge \bar{v}=\bar{\mu}$ or $\bar{\mu} \wedge \bar{v}=v$. Then (i) implies either $\bar{\mu} \leq \bar{\lambda}$ or $\bar{v} \leq \bar{\lambda}$.

Theorem 5.8. If the set of i-v fuzzy bi-ideals of a near-ring $N$ is totally ordered by inclusion, then each i-v fuzzy bi-ideal of $N$ is idempotent if and only if each i-v fuzzy bi-ideal of $N$ is prime.

Proof: Suppose each i-v fuzzy bi-ideal of $N$ is idempotent. Let $\bar{\lambda}$ be a arbitrary i-v fuzzy bi-ideal and $\bar{\mu}, \bar{v}$ be an i-v fuzzy bi-ideals of $N$ such that $\bar{\mu} \circ \bar{v} \leq \bar{\lambda}$. Since the set of i-v fuzzy bi-ideals of $N$ is totally ordered by inclusion, so either $\bar{\mu} \leq \bar{v}$ or $\bar{v} \leq \bar{\mu}$.

If $\bar{\mu} \leq \bar{v}$ then $\bar{\mu} \circ \bar{\mu} \leq \bar{\mu} \circ \bar{v} \leq \bar{\lambda}$, implies $\bar{\mu} \leq \bar{\lambda}$ as $\bar{\lambda}$ is semi-prime by Theorem 5.5. If $\bar{v} \leq \bar{\mu}$ then $\bar{v} \circ \bar{v} \leq \bar{\mu} \circ \bar{v} \leq \bar{\lambda}$, implies $\bar{v} \leq \bar{\lambda}$ as $\bar{\lambda}$ semi-prime by Theorem 5.5. So each i-v fuzzy bi-ideal of $N$ is prime.

Conversely, Suppose that every i-v fuzzy bi-ideal of $N$ is prime. Since every prime i-v fuzzy bi-ideal of $N$ is semi-prime. So by Theorem 5.5, each i-v fuzzy bi-ideal of $N$ is idempotent.

Theorem 5.9. For a near-ring $N$ the following assertions are equivalent:

(1) Set of i-v fuzzy bi-ideals of a near-ring $N$ is totally ordered by inclusion.

(2) Each i-v fuzzy bi-ideal of $N$ is strongly irreducible.

(3) Each i-v fuzzy bi-ideal of $N$ is irreducible.

Proof:

(1) $\Rightarrow(2)$ : Let $\bar{\lambda}$ be an arbitrary i-v fuzzy bi-ideal of $N$ and $\bar{\mu}, \bar{v}$ be i-v fuzzy biideals of $N$ such that $\bar{\mu} \wedge \bar{v} \leq \bar{\lambda}$. Since the set of i-v fuzzy bi-ideals of $N$ is totally ordered by inclusion, so either $\bar{\mu} \leq \bar{v}$ or $\bar{v} \leq \bar{\mu}$. Thus either $\bar{\mu} \wedge \bar{v}=\bar{\mu}$ or $\bar{\mu} \wedge \bar{v}=\bar{v}$, implies either $\bar{\mu} \leq \bar{\lambda}$ or $\bar{v} \leq \bar{\lambda}$.

(2) $\Rightarrow(3)$ : Let $\bar{\lambda}$ be an arbitrary i-v fuzzy bi-ideal of $N$ and $\bar{\mu}, \bar{v}$ be i-v fuzzy biideals of $N$ such that $\bar{\mu} \wedge \bar{v}=\bar{\lambda}$...(i)

Thus $\bar{\mu} \geq \bar{\lambda}$ and $\bar{v} \geq \bar{\lambda}$. (i) implies $\bar{\mu} \wedge \bar{v} \leq \bar{\lambda}$. So $\bar{\mu} \leq \bar{\lambda}$ or $\bar{v} \leq \bar{\lambda}$, as $\bar{\lambda}$ is strongly irreducible. Hence either $\bar{\mu}=\bar{\lambda}$ or $\bar{v}=\bar{\lambda}$. 


\section{V.Vetrivel and P.Murugadas}

(3) $\Rightarrow(1)$ : Let $\bar{\mu}$ and $\bar{v}$ be two i-v fuzzy bi-ideals of $N$ Then by Lemma 3.6, $\bar{\mu} \wedge \bar{v}$ is also i-v fuzzy bi-ideal of $N$ Also $\bar{\mu} \wedge \bar{v}=\bar{\mu} \wedge \bar{v}$, implies either $\bar{\mu}=\bar{\mu} \wedge \bar{v}$ or $\bar{v}=\bar{\mu} \wedge \bar{v}$. Thus $\bar{\mu} \leq \bar{v}$ or $\bar{v} \leq \bar{\mu}$.

\section{REFERENCES}

1. S.J.Abbassi and A.Z.Rizvi, Study of prime ideals in near-rings, Journal of Engineering and sciences, 2 (1) (2008).

2. S.Abou-Zaid, On fuzzy subnear-rings and ideals, Fuzzy Sets and Systems, 44 (1991) 139-146.

3. B. Davvaz, Fuzzy ideals of near-rings with interval valued membership functions, Journals of Sciences, 12 (2) (2001) 171-175.

4. S.D.Kim and H.S.Kim, On fuzzy ideals of near-rings, Bulletin of the Korean Mathematical Society, 33 (1996) 593-601.

5. A.Rosenfeld, Fuzzy groups, Journal of Mathematical Analysis and Application, 35 (1971) 512-517.

6. S.Bashir, Prime bi-ideals and strongly prime fuzzy bi-ideals in near rings, Annals of Fuzzy Mathematics and Informatics, to appear.

7. N.Thillaigovindan, V.Chinnadurai and S.Kadalarasi, Interval valued Fuzzy ideal of Near-rings, J. Fuzzy Math., 23 (2) (2015) 471-484.

8. T.Tamizh chelavam, N.Ganesan, On bi-ideals of near-rings, Indian J. Pure and Appl. Math., 18 (11) (1987) 1002-1005.

9. L.A.Zadeh, Fuzzy Sets, Information and Control, 8 (1965) 338-353.

10. L.A Zadeh, The concept of a linguistic variable and its application to approximate reasoning, Inform. Sci., 8 (1975) 199-249. 